EUROPEAN ORGANIZATION FOR NUCLEAR RESEARCH

\author{
CERN - PS DIVISION
}

PS/HP Note 99-11

Neutrino Factory Note-08

\title{
Transverse Collection of Pions
}

\author{
B. Autin, Ph. Royer
}

\begin{abstract}
The early stages of a neutrino factory are critical to fulfill physics requirements in the final muon storage ring. The transverse collection of pions behind the target is therefore a crucial feature for which different options exist. In this article, two collection systems using either quadrupoles or a transition solenoid between the target and the downstream channel are studied. The transition optics from the solenoid to a quadrupole channel is also discussed. Some figures of merit such as maximum transverse momentum captured or radial acceptance are compared as well as the technical challenges for each solution.
\end{abstract}




\section{Introduction}

Motivated both by the neutrino physics community and by the muon colliders collaborations, interest in a neutrino factory based on a muon storage ring has greatly increased recently [1]. The efficiency of a neutrino factory is highly dependent on the number of muons stored and therefore of the number of pions from which decay the muons through the well-known reaction:

$$
\pi^{ \pm} \rightarrow \mu^{ \pm} \nu
$$

This makes the focusing system which collects the pions behind the target one of the critical feature of the neutrino factory. This article focuses on this part of a whole scheme and compares various possibilities for collecting pions. It is therefore assumed that a given proton beam produced either by a linac or a synchrotron is impinging on a given target. Some numbers, such as production yields, may well depend considerably on the choice of the proton beam energy and of the type of the target, however this paper aims at defining and comparing some figures of merit of collection systems, for example the acceptance of the channel or the phase portrait of the beam.

\section{Magnetized target}

The common feature to solenoid and quadrupole capture is the use of a magnetized target. Whatever the type of target, it is immersed in a high solenoidal field which provides an appreciable shrinking of the phase space for the same maximum transverse momentum collected (see figure 1).
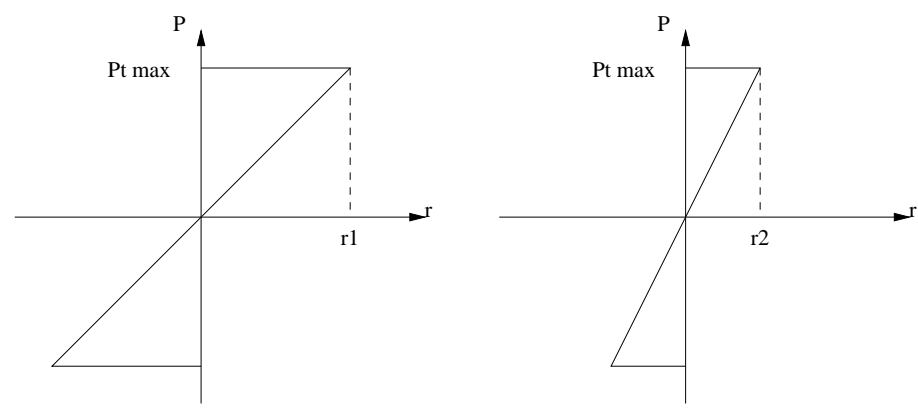

Figure 1: Phase space shrinking because of magnetized target 
The reduction of the physical dimensions of the pion beam is the result of the particles spiraling in the solenoidal field with a maximum radius half the radius of the solenoid. While particles produced with a maximum angle of $\theta$ outside any field would reach a radius $r_{1}=l \tan \theta$ after a typical target length $l$ of $12 \mathrm{~cm}$, inside the solenoid they are kept within the limit of the radius $r_{2}$ of the solenoid. For the following working values of $P_{\perp}=240 \mathrm{MeV} / \mathrm{c}$, a longitudinal rapidity of one and a solenoid of $8 \mathrm{~cm}$ radius, the ratio $r_{2} / r_{1}$ is 0.5 , i.e. the phase space is reduced by a factor one half.

Assuming that the particles are produced on the axis of the solenoid, the high field allows to collect pions with a large transverse phase space normalized acceptance

$$
A_{n}=\frac{r P_{\perp}}{m_{\pi} c}=\frac{e B_{0} r^{2}}{2 m_{\pi} c} \quad \text { with } \quad P_{\perp \max }=e B_{0} r / 2
$$

where $\mathrm{r}$ is the radius of the solenoid, $P_{\perp}$ the transverse momentum, $B_{0}$ the magnetic field on the axis of the solenoid and e the particle charge. Since we want $P_{\perp \max }$ to be maximum, we will choose $B_{0}$ maximum according to technical limits. Since $r$ is the physical radius of the initial pion beam escaping the target, it is determined by the requirements or limits of the downstream channel.

\section{$3 \quad$ Initial focusing}

Independently of the proton energy, the pion production integrated yields exhibit the same maximum steady curve in the rapidity range from one $\left(P_{\|}=\right.$ $165 \mathrm{MeV} / \mathrm{c})$ to two $\left(P_{\|}=500 \mathrm{MeV} / \mathrm{c}\right)$ on the longitudinal axis with a wide range of momenta in the transverse plane. The aim of the capture is to match the beam from the magnetized target to a realistic channel either using quadrupoles or solenoid collecting within this range of momenta.

\subsection{Interface conditions}

As stated above, the phase space at the target has a butterfly shape which is inscribed into an ellipse with the highest possible extension in transverse momentum and a small radius. Since the particles are spiralling inside the solenoid, the movement is coupled in both transverse planes and one has to consider the $4 \mathrm{D}$ canonical phase space $\left(x, p_{x}, y, p_{y}\right)$ where $p_{x}$ and $p_{y}$ are 
the canonical momenta. Indeed, at the end of the solenoid, the particules cross a varying solenoidal field and will therefore experience radial forces that will change their azimuthal momenta, hence their transverse momenta. However, the canonical momenta stay constant while crossing this end field [2]. Therefore, transforming the ellipses in the $4 \mathrm{D}$ canonical phase space $\left(x, p_{x}, y, p_{y}\right)$ into $\left(x, x^{\prime}, y, y^{\prime}\right)$ using $x^{\prime}=P_{\perp x} / P$ gives a symmetrical phase portrait in both transverse planes.

These interface conditions are valid not only directly behind the target but also along every solenoidal channel, one can choose to make the matching from the solenoid to the quadrupoles at different stages of the channel. We here study the two extreme cases.

\subsection{Direct quadrupole collection}

One can choose to collect the pions right behind the magnetized target using a standard quadrupoles channel. We are therefore in a phase space configuration where the collected transverse momentum must be as high as possible and the beam dimensions are small. In that case we are limited by the transverse angular acceptance of the following quadrupoles and to avoid high fields and large beams in these latter, we limit the transverse collection up to 50 $\mathrm{MeV} / \mathrm{c}$, that is to say a $20 \mathrm{~T}$ solenoid of radius $17 \mathrm{~mm}$, according to equation (1). For a rapidity equal to 1.5, the maximum angular acceptance is therefore $0.16 \mathrm{rad}$.
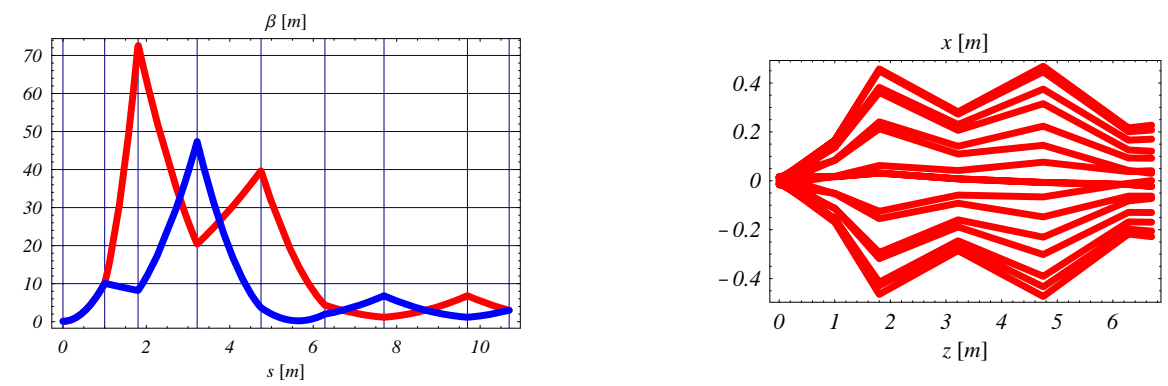

Figure 2: Quadrupole collection channel with thin lenses followed by a FODO cell and envelope tracking in the transverse horizontal direction at $P_{t o t}=300$ $\mathrm{MeV} / \mathrm{c}$. 
The beam is rotationally symmetrical in the transverse plane and the studied optics is made of a doublet which brings the beam from a symmetrical to an alternating configuration, followed by a triplet. The doublet brings the optical beta functions from a waist point to a crossover and the triplet reduces the beta functions so as to match the beam into a regular FODO cell which transports the beam while giving room enough to add RF cells between the quadrupoles.

The physical emittance

$$
\epsilon=\frac{r P_{\perp}}{P}
$$

is $2.8 * 10^{-3} \mathrm{~m}$.rad with $P=300 \mathrm{MeV} / \mathrm{c}$. The field gradient $G$ in a quadrupole of length $l$ and focal distance $f$ is given by $k=\frac{1}{f l}=\frac{e G}{p}$, and we can therefore draw the following list of parameters.

\begin{tabular}{||l|c|c|c|c|c||}
\hline Quadrupoles & Q1 & Q2 & Q3 & Q4 & Q5 \\
\hline $\mathrm{l}(\mathrm{m})$ & 0.3 & 0.5 & 0.4 & 0.4 & 0.3 \\
\hline $\mathrm{G}(\mathrm{T} / \mathrm{m})$ & 3.7 & 2.2 & 2.2 & 1.5 & 2.9 \\
\hline $\mathrm{B} \max (\mathrm{T})$ & 0.6 & 1.0 & 0.8 & 0.5 & 0.3 \\
\hline
\end{tabular}

Hence, as confirmed by tracking (see figure 2), the beam radius for $P=$ $300 \mathrm{MeV} / \mathrm{c}$ stays within the limit radius of $45 \mathrm{~cm}$ and the fields in the quadrupoles being less than $1 \mathrm{~T}$, they can be constructed with a standard technology. Nevertheless, one main difficulty is the large energy spread of the pions in the channel. Indeed, the optics design is made for the largest real emittance corresponding to the smallest energy and for higher energies, the emittance is reduced. However, this reduction of emittance does not compensate the fact that the optics is unadapted for different energies. As regards optics adaptation, the larger the energy spread the worst; so, simulations show larger beams for higher momentum particles launched in this channel. The standard way of correcting these chromatic effects is to introduce sextupoles and dispersion in the channel so that all particles see the same focalization, independently of their momentum. A bending magnets used to separate the proton beam from the pion beam at the very beginning of the collection might create the needed dispersion. From this point of view, a bending system could fulfill three roles: creating dispersion for sextupoles, separating the beams and shielding the rest of the channel. 


\subsection{Transition Solenoid}

Because of the limitation in the maximum transverse momentum collected with quadrupoles, one may think of using a transition solenoid between the target and the downstream quadrupole channel. Different systems may be considered, however an adiabatic system made of a slowly decreasing magnetic field over a few meters allows some larger radial and energy acceptances [3]. The main advantage of this kind of solenoids is their ability to transform a beam with large transverse momenta and small dimensions into a beam with smaller transverse momenta and larger dimensions. This solution has been retained by the american collaboration on muon colliders [4].

According to equation (1) and assuming a radius of $8 \mathrm{~cm}$, a field of 20 $\mathrm{T}$ and particle production on the axis, the maximum transverse momentum collected is $240 \mathrm{MeV} / \mathrm{c}$ and the normalized emittance

$$
\epsilon_{N}=\frac{r P_{\perp}}{m_{\pi}}
$$

is $0.14 \mathrm{~m} . \mathrm{rad}$. The maximum angular acceptance is deduced from $\sin \theta=$ $P_{\perp \max } / P$ which gives $\theta=0.96 \mathrm{rad}$ for the lowest longitudinal momentum of $165 \mathrm{MeV} / \mathrm{c}$, corresponding to a rapidity equal to one.

Let $\mathrm{z}$ be the coordonate of the axis of the solenoid, the field law is

$$
B(z)=\frac{B_{0}}{1+\alpha z} \quad, \quad \alpha=\frac{\epsilon B_{0}}{P_{0}}
$$

where $B_{0}=20 \mathrm{~T}$ is the field around the target, $P_{0}$ a central value of the scalar momentum and $\epsilon \ll 1$ a parameter of smallness. The system is described by two equivalent invariants:

$$
\begin{array}{cl}
\frac{P_{\perp}^{2}}{B} & \text { conservation of the magnetic moment } \\
B r^{2} & \text { conservation of the magnetic flux }
\end{array}
$$

both resulting from the invariance of the action integral under adiabatic changes [5]. These invariants allow to trace the transverse phase plane along the solenoid. If $a$ is the radius and $B_{s}$ the field of the solenoid at the end of the adiabatic device, the radial acceptance and the transverse acceptance at the entrance and at the output of the adiabatic device are respectively:

$$
\left\{\begin{array} { l } 
{ r _ { 0 } = \sqrt { \frac { B _ { s } } { B _ { 0 } } } a } \\
{ P _ { \perp 0 } = \frac { e B _ { 0 } r _ { 0 } } { 2 } = \frac { e \sqrt { B _ { s } B _ { 0 } } a } { 2 } }
\end{array} \quad \left\{\begin{array}{l}
r_{\text {final }}=a \\
P_{\perp \text { final }}=\frac{e B_{s} a}{2}
\end{array}\right.\right.
$$


where one can see the tranformation from large transverse momenta and small dimensions (from $P_{\perp 0}=240 \mathrm{MeV} / \mathrm{c}$ with $r_{0}=8 \mathrm{~cm}$ and $B_{0}=20 \mathrm{~T}$ ) to smaller transverse momenta and larger dimension $a=4 r_{0}=32 \mathrm{~cm}$ and $P_{\perp \text { final }}=\frac{P_{\perp 0}}{4}=60 \mathrm{MeV} / \mathrm{c}$ with $\left.B_{s}=1.25 \mathrm{~T}\right)$.

The Lagrangian equations written in the rotating Larmor frame can be integrated for this slowly varying magnetic field [3] and provide the following transport matrix for the position $\xi$ and canonical momentum $p_{\xi}$, decoupled in the rotating frame of angle the Larmor angle $\phi$ :

$$
\left(\begin{array}{c}
\xi \\
p_{\xi}
\end{array}\right)=\left(\begin{array}{cc}
\sqrt{\frac{B_{0}}{B}} \cos \phi & \frac{2}{e \sqrt{B_{0} B}} \sin \phi \\
-\frac{e \sqrt{B_{0} B}}{2} \sin \phi & \sqrt{\frac{B}{B_{0}}} \cos \phi
\end{array}\right)\left(\begin{array}{c}
\xi_{0} \\
p_{\xi 0}
\end{array}\right) \text { where } \phi=\int_{0}^{z} \frac{e B}{2 P} d z .
$$

If $R$ is the 4 by 4 rotation matrix of angle $\phi$ from the Larmor frame to a Cartesian frame, the matrices product $R M R^{-1}$ allows to track particles inside the adiabatic device in the $\left(x, p_{x}, y, p_{y}\right)$ frame and to plot the intersection of the $4 \mathrm{D}$-phase space volume with the plane $\left(y=0, p_{y}=0\right)$ in the initial and final configurations as done in figure 3 .
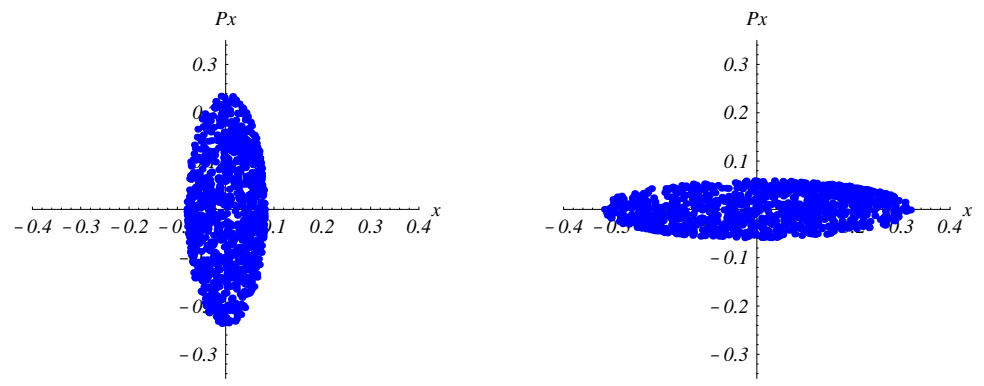

Figure 3: Transverse phase space evolution through the adiabatic device in one transverse plane from the input (target) to the output.

This new transverse phase space can be the beginning point for the transition to a quadrupole channel. The beta function is greater than before, $\beta=r^{2} / \epsilon=1.5 \mathrm{~m}$ with a physical emittance of $\epsilon=6.6 * 10^{-2} \mathrm{~m} . \mathrm{rad}$. Again, to match this optically symmetrical beam to an alternating gradient configuration, we use a doublet. However, since the emittance is huge because of the large acceptance of the adiabatic device, the beta functions have to be 
kept low to avoid large beam radii. Two schemes are proposed (figure 4). The first scheme is designed to transport the beam (for instance along the
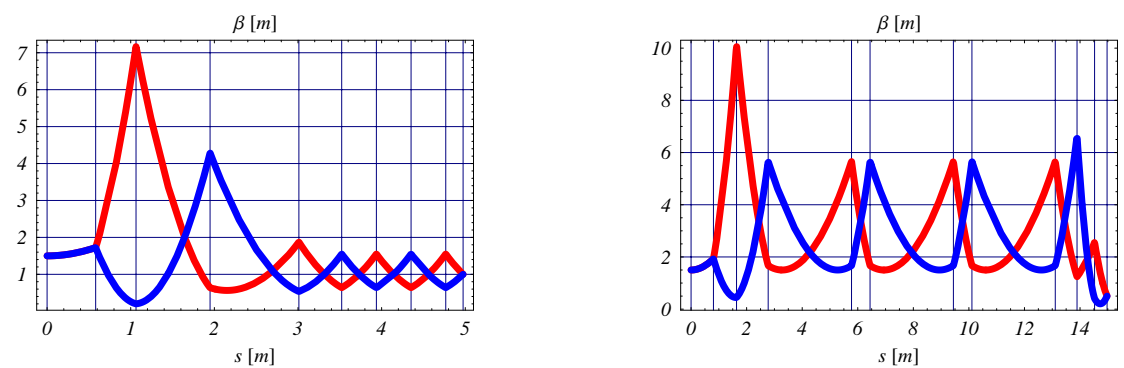

Figure 4: Transverse horizontal and vertical beta functions. The first scheme is designed to simply transport the beam; The second one allows to use RF in the channel.

drift between the two bunch rotations [6]). The initial doublet is followed by a second doublet which brings the beta functions down to $1 \mathrm{~m}$ into the FODO cells, each of total length $.8 \mathrm{~m}$. Here the maximum beam radius is reached in the second quadrupole of the doublet and is $.63 \mathrm{~m}$ for a pole tip field of $3.3 \mathrm{~T}$ which requires a superconducting device. In the FODO cells, for quadrupoles of $20 \mathrm{~cm}$ length, the pole tip field is $2.7 \mathrm{~T}$ for a maximum beam radius in the quadrupoles of $27 \mathrm{~cm}$ with a spacing left between the quadrupoles of the order of their length $(20 \mathrm{~cm})$.

The second solution drives the beta function to a higher value and therefore allows to use Collins insertions [7], which are a special kind of FODO cells giving more spacing between the quadrupoles. This option is valid if one needs room to put RF cavities between quadrupoles either to accelerate pions or to make a bunch rotation. In that case, the cavities are free from any magnetic field between the quadrupoles, and superconductive devices are then conceivable. The conterpart is a wide beam with maxima of $54 \mathrm{~cm}$ radius in the quadrupoles and minima of $31 \mathrm{~cm}$ of radius in the middle of the drift. Cavities must therefore be chosen to cope with a limited longitudinal space of roughly $2.5 \mathrm{~m}$ and large acceptances. In the Collins insertions, the quadrupoles are $20 \mathrm{~cm}$ long for a pole tip field of $2.6 \mathrm{~T}$, which requires superconducting magnets. The space left between quadrupoles is then more than $40 \mathrm{~cm}$ which doesn't bring any fringe fields problems. At the end, the beta functions are driven back to small values by a doublet, allowing for example 
the matching to standard but compact FODO cells.

Although the effect of the large energy spread remain to be studied in those channels, an interesting alternative to a long, big solenoid might emerge from these two schemes. In both cases the space between the quadrupoles should be larger than the inner diameter of the quadrupoles. The schemes shown here are thus limited in acceptance.

\section{Conclusion}

We have compared the figures of merit of two possible scenarios for the transverse collection of pions and seen that, in the first few meters behind the target, the variety of strategies requires to be carefully studied in order to find the optimum solution.

The magnetized target is undoubtedly an advantage since it reduces the phase space emittance to be carried by the downstream channel.

The direct quadrupole collection remains attractive by its simplicity. However the number of particles captured may not reach the high requirements of particle physicists for the final storage ring, because of the limitation in transverse momentum. This could be partly solved by increasing the amount of particle in the transverse momentum range $0-50 \mathrm{MeV} / \mathrm{c}$ by increasing the power on the target up to unprecedented ranges of power. The wide energy spread inside the channel causes the optics to be clearly inadapted for particule of different momenta and gives rise to large beam radii. Introducing dispersion and using sextupoles to correct this effect and to make a focalization identical for all particules is an option to be studied.

On the contrary, the capture using an adiabatic device in the few meters seems promissing since all particules up to a transverse momentum of 240 $\mathrm{MeV} / \mathrm{c}$ are carried away inside a decreasing magnetic field. In that scheme, the large transverse acceptance at the beginning is transformed into a large radius beam at the end of the capture. Then, one option is to continue the transport in a big solenoid until cooling and the reduction of transverse dimensions. We have here studied an alternative to that proposal which is the transition from the capture solenoid to a quadrupole channel. Two schemes were presented: the first one increases the beta function to give room enough to use RF cavities outside of the magnetic field (although with large acceptance), while the other may be used to transport the beam over a long drift with a compact FODO cell. In both cases, the beam is large, 
and so are the magnetic components. Transverse cooling is thus necessary to reduce the excessive beam emittance $[6,8]$.

Either with the solenoid capture or with a quadrupole channel, the question of separating the primary proton beam from the pion beam still need to be addressed.

\section{References}

[1] B. Autin, A. Blondel, J. Ellis, "Prospective study of muon storage rings at Cern", Cern Yellow Report 99-02, 1999.

[2] R.Chehab, private communication.

[3] R.Chehab, "Positron sources", LAL/RT 92-17 (1992).

[4] R.B. Palmer et al., "Muon colliders", Proceedings of 9th Advanced ICFA Beam Dynamics Workshop, 1995, Montauk, NY, pp.3-30 (1996).

[5] Martin Reiser, "Theory and Design of Charged Particle Beams", Wiley series in beam physics and accelerator technology, Wiley, New-York, 1994.

[6] R.B. Palmer for the collaboration, "Draft Parameters of a Neutrino Factory", Muon Collider Note 46, version 9/3/99.

[7] B. Autin (editor), C. Carli, T. D'Amico, O. Grobner, M. Martini, E. Wildner, "BeamOptics A Program for Analytical Beam Optics", Cern Yellow Report 98-06, 1998.

[8] C.Johnson, E. Keil, R. Palmer, " A Cost Effective Design for a Neutrino Factory", These proceedings. 